\title{
Vantagens da aglomeração: reunindo percepções e perspectivas
}

Advantages of agglomeration: gathering perceptions and perspectives

\author{
${ }^{1}$ Esdras Kênia de Paula
}

1 Mestre em Políticas Públicas, Estratégia e Desenvolvimento pelo Instituto de Economia - UFRJ. Especialista em Gestão Pública Municipal - UFF. Graduada em Administração de Empresas - UFF.

\section{RESUMO}

Este artigo propõe-se a tratar das vantagens da aglomeração para o desenvolvimento econômico local, frente às demandas globais cada vez mais dinâmicas. Reúne a visão de alguns autores clássicos como Marshall, até outros contemporâneos como La Rovere, com uma visão mais centrada em Economia da Inovação. Nessa perspectiva, tratamos a construção temporal, dos caminhos que levaram o conhecimento aliado à inovação nas empresas e a importância dos seus resultados para a economia local, que tomaram a dimensão atual para o desenvolvimento econômico. Encerramos o estudo com algumas conclusões acerca das vantagens das aglomerações para o desenvolvimento local e econômico, com algumas perspectivas.

\section{PALAVRAS-CHAVE}

Inovação; conhecimento; inovação; desenvolvimento econômico local.

\section{ABSTRACT}

This article proposes to deals with the agglomeration advantages for local economic development front to global demands increasingly dynamic. It brings together the vision of some classic authors like Marshall to other contemporaries as La Rovere, with a more focused on Innovation Economy vision. In this perspective we treat the temporal construction of roads leading knowledge combined with innovation in companies and the importance of its results for the local economy, taking the current dimension to economic development. We ended the study with some conclusions about the benefits of agglomeration for local and economic development, with some perspective.

\section{Como você deve citar?}

PAULA, E. K. Vantagens da aglomeração: reunindo percepções e perspectivas. Cadernos UniFOA, Volta Redonda, $n^{\circ}$ 25, quadrimestral, p. 69-76, ago. 2014. 


\section{INTRODUÇÃO}

Nos debates acerca das questões relacionadas à aglomeração local é notória a intensiva importância, em nível mundial, de tais aglomerados para o desenvolvimento econômico. Especialmente a partir da década de 1970, "a produção tem se tornado mais intensiva em conhecimento, na medida em que o investimento em intangíveis [...] adquiriram maior importância na produção de bens e serviços" (Mytelka \& Farinelli, 2005, p. 347). Dessa forma, o foco dado no conhecimento deu-se em todos os setores, até mesmo nas indústrias tradicionais, também conhecidas como de baixa tecnologia, em face de um novo cenário econômico globalizado, com grandes corporações, interagindo mutuamente, com vultoso crescimento internacional e sistemas de comunicação mais flexíveis. Assim os esforços passaram a se concentrar na produção intensa de conhecimento, onde o mercado se tornou imperativo na capacidade de inovar das empresas, visto que "o processo de geração e difusão das inovações seria o principal fator determinante dos chamados ciclos longos do capitalismo" (La Rovere, 2006, p. 285).

Nesse sentido, tem-se uma mudança em relação à teoria econômica convencional, no que tange o sentido da adoção de inovações por parte das empresas:

\footnotetext{
Dado um determinado fluxo de inovações, sua adoção irá depender do ambiente competitivo da empresa, que é um ambiente seletivo, uma vez que a empresa irá decidir a respeito da adoção de inovações, tendo em vista a taxa de lucros praticada no ramo industrial no qual ela se situa, das condições do investimento e das condições de imitação das inovações. Além disso, características institucionais - tais como o financiamento às atividades de pesquisa e desenvolvimento (P\&D) e a estrutura da indústria, afetam as condições de geração e de adoção de inovações. Considera-se assim a existência um processo de feedback constante na atividade inovadora, o qual anula a decisão de seus determinantes em fatores de oferta e demanda (LA ROVERE, 2006, p. 286).
}

Assim podemos notar que, a decisão de adotar ou não determinadas atividades inovadoras depende não só de fatores externos ao ambiente empresarial, mas também de fatores endógenos à dinâmica empresarial, bastante pautado no conhecimento constituído por cada uma conjuntamente com o ambiente na qual está inserida.

Este trabalho se propõe a percorrer esses caminhos construídos através do conhecimento aliado à inovação e às tipologias industriais desenvolvidas a partir de novas demandas e percepções, quanto à dinâmica do ambiente industrial e seus diferentes elementos que compõem as aglomerações, procurando demonstrar a importância desta para o crescimento econômico e desenvolvimento intra e interaglomerações. Para tanto, baseamo-nos, especialmente, nos seguintes trabalhos para desenvolver essa discussão: La Rovere (2006), Markusen (1996) e Mytelka \& Farinelli (2005). Foram citados, ainda, outros autores a titulo de complementação para um melhor entendimento dos conceitos abordados.

\section{VANTAGENS DA AGLOMERAÇÃO}

Como foi apresentado, diante de um cenário cada vez mais dinâmico e competitivo, onde os esforços para a manutenção dos mercados tendem a se ampliar, nos surge o seguinte questionamento: Por que determinados lugares são capazes de sustentar a atratividade de capital e trabalho, tornandose ainda vantajosos e diferenciados?

Markusen (1996) nos sugere que, alguns lugares, considerados como "pegajosos", conseguem ancorar atividades geradoras de renda, dadas suas condições de produção e reprodução. Para tanto, esses "lugares pegajosos" precisam alcançar condições de produção local e reproduzir seus gastos ao mínimo possível. A autora explica esse modelo de sucesso através dos New Industrial Districts, baseado 
no modelo de expansão de indústrias bem sucedidas na Itália. Sua aceitabilidade se deve ao sucesso que algumas empresas inovadoras conseguiram obter dentro de um sistema regional de governança cooperativa industrial, que se adaptaram mesmo com tendências globalizantes, contudo tais tendências tornam a produção nesses lugares cada vez mais escorregadia, devido à hipermobilidade do trabalho e da reprodução. O New Industrial Districts é considerado uma nova forma de paradigma emergente, pois são teoricamente plausíveis e empiricamente demonstrados. Como atributos de sucesso desses lugares a autora destaca a aceleração e integração do mercado mundial rentável.

Para destacar a importância dessa concepção de paradigma, Khun (1962), autor do termo "paradigma", demonstra que a história da ciência não é linear e cumulativa, tendo como característica fundamental a interruptividade que se dá num contexto social científico, ou seja, numa comunidade científica. A cada ruptura emerge um novo paradigma e essa troca depende da aceitação dos cientistas, gerando certa inércia à ciência. Esse paradigma é entendido como um modelo padrão a ser seguido, complexo por trazer a definição de um novo problema e um conjunto de soluções para o mesmo. Por isso o paradigma tem um caráter normativo, pois tem por finalidade apresentar para os cientistas a solução para novos problemas. Essa mudança de paradigma é que dá origem as Revoluções Científicas, que são entendidas como situações que transbordam as instituições, sendo necessário criar soluções que satisfaçam a esse novo paradigma vigente.

Tem-se que, desde Marshall, as aglomerações são tidas como forças motoras para o desenvolvimento, identificando-as nas formas originais dos distritos industriais da Itália , porém de acordo com sua formulação, o distrito industrial vislumbra uma região onde os negócios são compostos por estruturas pequenas, com decisão de investimento e produção local; as transações são entre compradores e vendedores, firmando-se apenas por longos termos de contratos e compromissos. Marshall não reconhece o estabelecimento de ligações com firmas foras do distrito, pois, segundo ele, o que faz do distrito industrial vibrante são a natureza e a qualidade do mercado de trabalho local, fatores internos ao distrito e altamente flexíveis.

\footnotetext{
Somente mais tarde, depois da intensificação do dinamismo global, como mencionado anteriormente, foi que alguns estudiosos dos processos de industrialização retornaram suas pesquisas às buscas de aglomerações inovativas, como aquelas de Marshall, em outras partes do mundo, na tentativa de identificar laços esforçados inter e entraglomerações. Seguindo essa lógica, Markusen identifica três outros tipos de distritos industriais: In probing the conditions under which some manage to remain "sticky" places in "slippery" space, this paper rejects the "new industrial district", in either its Marshallian or more recent Italianate form, as the dominant paradigmatic solution. I identify three additional types of industrial districts, with quite disparate firm configurations, internal versus external orientations, and governance structures: a hub-and-spoke industrial district, revolving around one or more dominant, externally oriented firms; a satellite platform, an assemblage of unconnected branch plants embedded in external organization links; and the state-anchored district, focused on one or more public-sector institutions (MARKUSEN, 1996, p. 293).
}

Dessa forma, a autora rejeita a tipologia de distritos Marshalianos como sendo a solução de paradigma dominante, a partir do seu trabalho, o qual identifica outros três tipos de distritos industriais que fazem frente à dinâmica econômica de modo eficaz, respondendo a demandas cada vez mais amplas onde, "enfatiza-se o processo no qual empresas, em interação umas com as outras e apoiadas por diferentes instituições e organizações [...] desempenham um papel-chave adequando novos produtos, novos processos e novas formas de organização" (Mytelka \& Farinelli, 2005, p. 348). Neste trabalho houve também um esforço na tentativa de identificar se existem aglomerações que são "flexibly specialized" nos três modelos de alternativas desses lugares pegajosos analisados. 
De acordo com este estudo, os autores da área não têm estabelecido rigorosamente quais são as ferramentas paras essas novas alternativas de distritos industriais, em caminhos que permitam o fácil acesso de suas coincidências e crescimento no espaço e no tempo, ou seja, da aderência de tais lugares. Temos que os limites da flexibilidade especializada dessas novas tipologias constituem uma nova forma de paradigma emergente, dados os atributos que fazem com que esses "lugares escorregadios" sejam bem sucedidos, dentre eles está à habilidade de integração que suas aglomerações exibem.

Uma vez que as fontes de rentabilidade tendem a variar ao longo da maturação de uma indústria e estão relacionadas à mudança na forma de competição, estruturas organizacionais, características ocupacionais e tendências locacionais, a vultuosidade da habilidade de integração na dinâmica do desenvolvimento de longo prazo, juntamente com outros constituintes presentes nos distritos, são determinísticos no que concerne à resiliência e vulnerabilidade de atrofia em longo prazo.

Neste sentido, relaciona-se ainda à provável trajetória e competitividade futura do conjunto industrial existente à sua capacidade de liberar recursos ancorados localmente para novos setores especializados não relacionados, extrapolando os limites de enraizamento das empresas dentro de seus distritos, formando redes não locais, cuja capacidade inovativa e conhecimento se destacam.

\section{A IMPORTÂNCIA DO CONHECIMENTO E DA INOVAÇÃO PARA OS AGLOMERADOS}

Conforme observado por Mytelka \& Farinelli (2005), todo tipo de aglomeração oferece oportunidades únicas de engajamento com uma ampla gama de ligações que estimulam o aprendizado e a inovação, que é necessária para a transformação de indústrias tradicionais que, por sua vez, são consideradas o ponto crítico de acordo com a perspectiva da inovação, pois esse setor demanda a formulação e adoção de políticas diferenciadas relacionadas ao aprendizado e seu processo de implementação, dada sua importância, uma vez que contribuem significativamente para o crescimento econômico. Além disso, "as indústrias tradicionais são plataformas em potencial para catching up por parte dos países em desenvolvimento. Mas para que isso ocorra, os formuladores de políticas terão de adotar uma perspectiva das oportunidades para aprendizado e inovação [...]" (2005, p. 371).

A estabilidade nessas relações e a colaboração entre os agentes podem resultar em eficiências coletivas, como redução de custos de transação, aceleração da inovação, redução de conflitos e meIhoria no ingresso ao mercado ${ }^{2}$. Além disso, as aglomerações geram externalidades positivas ${ }^{3}$ como: a disponibilidade de mão de obra qualificada, de determinados tipos de infraestrutura, trocas informais geradoras de inovação e do aprendizado possibilitado pela adoção de convenções ${ }^{4}$. As instituições políticas e sociais têm papel fundamental no desenvolvimento dessas atividades de parceria e no estímulo à produção em todos os níveis.

A partir do momento que os governos - seja na esfera municipal, regional ou nacional - passaram a considerar as vantagens decorrentes da localização como fundamentais para o desenvolvimento, começaram a promover mecanismos de aglomeração. Processo crescente, já no inicio dos anos 1970, o paradigma fordista começa a entrar em crise e, na década de 1980, estudava-se sistemas de aglomeração com foco nas pequenas e médias empresas. Devido à alta pressão competitiva instalada

\footnotetext{
2 Schmtiz (1997) apud Mytelka \& Farinelli (2005).

Segundo os estudos sobre a Teoria da Firma, o conhecimento gerado internamente é considerado um ativo que entra no processo produtivo. Isso muda a visão de competitividade, pois implica em não trocar com muita freqüência os recursos, visto que gera custos de produção e de transação porque internamente se constrói uma rede de relacionamento.

3 Paci \& Usai (2000) nos alerta que as externalidades podem ser também negativas, pois tanto podem aglomerar como podem afastar pessoas.

4 Storper, 1995; Maskell, 1996.
} 
mundialmente por causa, entre outros motivos, dos choques do petróleo e a falência dos ganhos de escala, consequentemente, houve uma mudança de paradigma "caracterizado pela incorporação de novas tecnologias organizacionais a um ritmo e abrangência sem precedentes na história econômica" (TIGRE, 1998, p. 88). Algumas mudanças decorrentes da transição entre os paradigmas foram resumidas, conforme podemos observar.

Quadro 1 - Mudanças do paradigma fordista para o das tecnologias da informação

\begin{tabular}{|l|l|}
\hline Paradigma fordista & Paradigma das tecnologias da informação \\
\hline Intensivo em energia & Intensivo em informação \\
\hline Padronização & Customização \\
\hline Mix de produtos estável & Rápidas mudanças no mix de produtos \\
\hline Produtos com serviços & Serviços com produtos \\
\hline Firmas isoladas & Redes de firmas \\
\hline Estruturas hierárquicas & Estruturas horizontais \\
\hline Departamental & Integradas \\
\hline Centralização & Inteligência distribuída \\
\hline Especialização & Polivalência \\
\hline Planejamento & Visão \\
\hline Controle governamental & Papel do governo: informação, coordenação e regulação \\
\hline
\end{tabular}

Fonte: Tigre (1998, p. 89).

A partir de então, de acordo com La Rovere (2006), as empresas passaram a interagir em mercados globais, em busca de uma produção mais flexível e tentando diversificar seus produtos. Tal busca levou à formação de redes de empresas relacionadas a seus produtos, visando assegurar seus clientes, de forma análoga. Podemos relacionar essa nova dinâmica à tipologia dos New Industrial Districts, apresentado pela autora Ann Markusen, que afirma que, embora algumas economias cresçam sob a presença de distritos industriais marshalianos, a ascendência paradigmática dessa nova forma do espaço industrial, mais acelerado e igualmente interativo, descreve a experiência da maioria dos aglomerados de rápido crescimento nos países industrializados e naqueles em industrialização. Cabe ressaltar que essa experiência foi expandida também com o propósito de conter a desindustrialização e reter empregos nas pequenas e médias empresas e não só com o objetivo de descrever novos espaços industriais e suas novas formações aglomerativas. Na verdade, é mais uma mistura de distritos que um puro caso de distrito industrial italiano, na sua forma original.

Geralmente grandes economias ${ }^{5}$ têm dificuldades para encontrar uma única instância de distrito industrial que seja flexivelmente especializada ao ponto que possa representá-las e na qual possa se desenvolver. Assim as grandes metrópoles apresentaram um crescimento baseado no desempenho de firmas sustentadas pelas tipologias: distrito centro-radial (hub), distritos ancorados pelo Estado ou plataforma industrial satélite, ou ainda, por uma combinação dos três tipos, o que foi normalmente constatado, pois cada conjunto de cidades possui suas características próprias, por contar com diferentes tecnologias, universidades, instalações, habilidades de interação, presença do estado de

5 As apontadas por Markusen foram: Coreia do Sul, Japão, Brasil e Estados Unidos, sendo que este último teve suas experiências baseados na tipologia de distritos industriais marshalianos até mesmo com sua nova versão italiana. 
bem-estar social e capacidade de mobilização, condições em que essas metrópoles puderam alimentar até mesmo o crescimento em seus arredores, gerando assim externalidades.

À medida que tal processo se desenvolve, os aglomerados transformam-se de simples espaços aglomerativos em sistemas de inovação dinâmicos, onde os fluxos, sejam eles de trabalho, capital, tecnologias, se articulam mais rapidamente. Segundo Amin \& Cohendet (2005), o sistema setorial de inovação ocorre até mesmo com empresas localizadas distantes uma da outra, pois a importância da proximidade é tanto geográfica quanto relacional. Os autores estabelecem ainda os elementos do sistema de inovação, através da Triple Helix. São eles: empresas, governo e universidades, que interagem para produção do conhecimento. Esses aglomerados são consideravelmente diversificados, construindo trajetórias de desenvolvimento singular, pois neles convivem elementos tipológicos diferentes por absorverem realidades distintas, até o ponto em que as demandas de uma dada realidade não são mais respondidas pelo paradigma vigente, como já foi visto, sendo necessário um novo paradigma:

\footnotetext{
Na medida em que cada fator-chave requer uma nova infra-estrutura facilitadora e pode introduzir mudanças organizacionais, há períodos nos quais diferentes paradigmas coexistem. Além disso, um novo paradigma não apenas propicia o surgimento de novas indústrias como renova e transforma as indústrias existentes. Um novo paradigma redefine desta forma as condições de competitividade das empresas e cria oportunidades para países em desenvolvimento reduzirem o hiato tecnológico que os separa dos países desenvolvidos. [...] À medida que as inovações associadas ao novo paradigma se difundem, os agentes econômicos vão se apropriando dos ganhos associados a essas novas inovações, mediante a acumulação de conhecimento tácito, e protegendo seus ganhos recorrendo a medidas de proteção intelectual (La Rovere, 2006, p.294-5).
}

Com relação à acumulação e disseminação de conhecimento tácito, Gertler (2003) chama atenção para a importância do contexto geográfico para a construção do conhecimento e revela que sua produção ainda está na metrópole, pois a reprodução do conhecimento depende de interação e proximidade física, organizacional e relacional, como já foi ressaltado também por Amin \& Cohendet (2005). 0 conhecimento tácito pode estar presente em relações globais, construindo redes, através das inúmeras fontes de tecnologia, desde que o procedimento adotado se torne uma rotina, até o momento que esse conhecimento se torne uma vantagem competitiva.

\section{CONCLUSÕES}

Conforme argumentado, vemos que os processos aglomerativos sempre tiveram um papel fundamental no crescimento econômico e no desempenho dos sistemas sustentados por ele, como é o caso das indústrias. Destaca-se a inovação no cerne do desempenho das indústrias propiciada pela aglomeração.

Voltando ao nosso questionamento inicial: por que determinados lugares são capazes de sustentar a atratividade de capital e trabalho, tornando-se ainda vantajosos e diferenciados? Podemos concluir que a atratividade desses lugares, na verdade, é composta por forças variadas e complexas que, interagindo conjuntamente, são capazes de ser bem sucedidos por não se concentrarem apenas em instituições locais e comportamentais, embora se constituam aglomerados, pois estão inseridos em um meio externo, onde de articula uma dinâmica paradigmática baseada em uma dada realidade que é mutável.

As inúmeras vantagens da aglomeração é outro fator de evidência para responder tal questionamento. Alguns pontos merecem destaque: facilita a produção e disseminação de conhecimento tácito; permite um aprofundamento da base local de conhecimento nas indústrias tradicionais; possibilitam 
habilidades de interatividade entre e interaglomerados, permitindo assim a formação de redes não locais; permitem criação de vantagem competitiva dentro dos aglomerados; a colaboração dos agentes gera eficiência coletiva que extrapola os limites do aglomerado; estimulam o aprendizado e a inovação; geram externalidades positivas; propiciam proximidade de modo a compartilhar conhecimento e inovação.

A autora Ann Markusen, salienta a importância dos distritos, através da formulação dos New Industrial Districts, considerado uma nova forma de paradigma emergente, no tocante à tipologia de distritos industriais sendo eles: distrito centro-radial (hub), distritos ancorados pelo Estado e plataforma industrial satélite, além do original distrito industrial marshaliano, identifica o conceito de flexibly specialized, característica dos novos distritos analisados que exibem alta habilidade de integração em suas aglomerações.

Markusen (1996, p. 310) sugere algumas estratégias para melhorar a concorrência regional e as taxas de crescimento diferenciado para os aglomerados, a saber: fazer um esforço para determinar quantos distritos de cada tipo a economia nacional poderia sustentar; desenvolver uma estratégia para estabilizar distritos existentes, canalizando os novos para as zonas deficitárias; proibir o uso de fundos públicos para subsidiar a concorrência entre regiões; e monitorar e, se necessário, alterar política pública nacional com implicações regionais substanciais, tais como descentralização do poder e responsabilidade aos níveis subnacionais, os novos regimes comerciais, iniciativas de políticas macroeconômica, mercado financeiro estruturado, desenvolvimento para o terceiro mundo, trabalho internacional e direitos humanos.

A partir desses esforços, canalizando todo o aprendizado gerado e o intercâmbio de conhecimento oriundos dos aglomerados, tem-se como resultado sistemas de inovação que tendem a ampliar os elos da indústria em novas bases de conhecimento, onde é imperativo descobrir modos de inovar para sustentar novos processos. Nesse sentido, é valido ressaltar o papel que políticas bem elaboradas e estruturas de apoio têm ao estimular novos hábitos e práticas, de forma a criar conexões mais amplas para o desenvolvimento e crescimento econômico.

Finalmente, a natureza na mudança de paradigma leva a uma nova compreensão no sentido das aglomerações a sistemas de inovação em âmbito global, pois os propósitos, para se aglomerar, e o tipo de relacionamento entre esses sistemas, mudam numa perspectiva da dinâmica industrial, conforme ocorreu entre os anos de 1970-80, em relação ao modo que se dava na concepção de distritos industriais de Marshall, onde as ligações entre as capacidades dos aglomerados e essa dinâmica precisam ser exploradas para que haja uma renovada tendência à capacidade inovativa. 


\section{REFERÊNCIAS}

AMIN, A. COHENDET, P. Geographies of knowledge formation in firms. Industry and Innovation. Vol. 12, n.4, 465-486, Dezembro 2005.

ARMSTRONG, H. ; TAYLOR. J. Regional Economics and Policy. 3rd edn. Oxford: Blackwell Publishers, pp. 149-168, 2000.

COOKE, P., BOEKHOLT, P. and TO“ DTLING, F. The Governance of Innovation in Europe: Regional Perspectives on Global Competitiveness. London: Pinter. 2000.

DESROCHERS, P.; HOSPERS, G.J. Cities and the Economic Development of Nations: An Essay on Jane Jacobs'Contribution to Economic Theory. Canadian Journal of Regional Science XXX, 1, 115-130, 2007.

DOSI, G. The Nature of the Innovative Process. In.Dosi, G.; Freerman, C.; Nelson, R; Silverberg, G. \& Soete, L. (eds.). Technical Change and Economic Theory. Londres: Pinter, 1988.

FAURÉ, Y. e HASENCLEVER, L. Caleidoscópio do desenvolvimento local no Brasil: diversidade nas abordagens e na experiência. Rio de Janeiro: E-Papers, 2007.

GERTLER, M.S., Tacit knowledge and the economic geography of context, or the undefinable tacitness of being (there). Journal of Economic Geography 3, 2003, p.75-99.

KHUN, T. A Estrutura das Revoluções Científicas. São Paulo: Ed. Perspectiva, 1962.

KRUGMAN, P. Increasing Returns and Economic Geography. The Journal of Political Economy, Vol. 99, No. 3. (Jun., 1991), pp.483-499. 1991.

LA ROVERE, R. L. Paradigmas e Trajetórias Tecnológicas. In: Pelaez, V.; Szmrecsanyi, T. (org.) Economia da Inovação Tecnológica. São Paulo: Hucitec, 2006.

LA ROVERE, R. L.; SHEHATA, L. D. Políticas de apoio a micro e pequenas empresas e desenvolvimento local: alguns pontos de reflexão. Redes, Santa Cruz do Sul, vol. 11 n. 3, p. 9-24, 2006.

MARKUSEN, A. Sticky places in slippery space: A typology of industrial districts Economic Geography. 72 (3), jul. 1996, p.293-313.

MYTELKA, L.; FARINELLI, F. De Aglomerados Locais a Sistemas de Inovação. In LASTRES, H.M.M et al. (org.) Conhecimento, Sistemas de Inovação e Desenvolvimento. Rio de Janeiro: Ed. UFRJ/Contraponto, 2005.

$\mathrm{PACl}, \mathrm{R}$. USAI, S. Externalities, knowledge spillovers and the spatial distribution of innovation. Geojournal, vol.4, 2000.

TIGRE, P.B. Inovação e Teorias da Firma em 3 paradigmas. Disponível em: http://www.ie.ufrj.br/oldroot/ hpp/intranet/pdfs/inovacao_e_teorias_da_firma_em_tres_paradigmaspaulotigre.pdf. 